\title{
Insecticide resistance studies of Cypermenthrin 25EC and Chlorpyriphos 20EC against Spodoptera litura fabricius, 1775 (Lepidoptera: Noctuidae)
}

\author{
Rupa Sule and Dolly Kumar* \\ Department of Zoology, Faculty of Science, \\ The Maharaja Sayajirao University of Baroda, Vadodara-390 002, Gujarat, India. \\ Received: 30-03-2019 \\ Accepted: 24-05-2019
}

DOI: 10.18805/IJARe.A-5260

\begin{abstract}
Spodoptera litura is one of the important pests and causes considerable damage to the crop. The indiscriminate use of insecticides result in development of resistance. Hence experiments were planned in laboratory conditions. The pest was reared in laboratory for many generations without exposure to any insecticides during 2018-19. After ensuring infection free culture, the insecticide resistance monitoring assays were performed to determine the development of resistance against two insecticides Cypermethrin 25EC and Chlorpyriphos 20EC by leaf-dip methodology. It was performed for five subsequent generations. The experimental results indicate greater resistance developed in Cypermehtrin 25EC @ 0.5 ppm (LC 50: 0.49, LC 90: 0.94) as compared to Chlorpyriphos 20EC @ 0.05 ppm. (LC 50: 0.05, LC 90: 0.34). This shows that there is onset of resistance in Cypermethrin 25EC, which may cause adverse effects, if not used in proper rotation with other insecticides.
\end{abstract}

Key words: Chlorpyriphos, Cypermethrin, Insecticides, Laboratory, Resistance, Spodoptera litura.

\section{INTRODUCTION}

Indiscriminate pesticide use is detrimental to the environment and human health and increases insects' resistance to pesticides (Ramasamy, 2012). Previous exposure with insecticides can confer resistance to newly introduced insecticides through cross-resistance reducing the effectiveness of new insecticides (Rehan et al., 2011). The problem of development of resistance to insecticides is more acute in Spodoptera litura (Fabricius) (Lepidoptera: Noctuidae) because of its polyphagous nature and rapid multiplication (Ramakrishnan et al., 1984). The current study is carried out on laboratory culture of Spodoptera litura. This pest was brought from the nearby fields of Vadodara, Gujarat. S. litura has been shown to be resistant to a wide range of insecticides, which has led to sporadic outbreaks of the pest and failure of crops (Ahmed et al.,2007). It is recognized as a serious cosmopolitan pest with considerable host range of economically agricultural crops such as cotton, groundnut, soybean, tomato and many other crops (Hiroichi et al., 1997). The presence of this pest on different crops throughout the year has widely exposed it to insecticides and resulted in the rapid development of resistance to a range of insecticides (Ahmed et al., 2008). The tobacco caterpillar, Spodoptera litura is an agriculturally important pest species. The management of the pest has therefore become increasingly difficult all over the world and the most commonly used insecticides are ineffective in controlling it (Tong et al., 2013). The insecticides are classified into various groups according to the toxicity levels i.e extremely toxic, highly toxic, moderately toxic and slightly toxic. Hence keeping in mind the indiscriminate use of various types of insecticides and toxicity levels conferred by insecticides, this study has been planned to observe the effect of two insecticides. Commercial formulations of insecticides used in this experiment were: Auzar® (Cypermethrin 25\% EC, Biostadt) and Dursban ${ }^{\circledR}$ (Chlorpyriphos 20\%EC, Dow Agrochemicals). Both these insecticides have greater effectiveness against all lepidopteran pests. Spodoptera litura is an indigenous pest of a variety of crops in South Asia and was found to cause $26-100 \%$ yield loss in groundnut (Dhir et al., 1992). The Indian agricultural sector has undergone tremendous transformation over the years from a state of severe food crisis to self-sufficiency in food grain production (Priscilla et al.,2017). Following the reports of various insecticides which are sprayed by farmers in fields, this work was planned as a laboratory study in controlled environment. This study is expected to be fruitful in implementing integrated pest management strategies. It will prove to be a helpful data for the agriculture professionals as well as scientists working towards a common cause of resistance development in pests like Spodoptera litura. Hence the purpose of this study is to take into account the development of resistance of Spodoptera litura a major pest of agricultural crops.

\section{MATERIALS AND METHODS}

Collection and preservation: A site survey was done in some parts of Vadodara, Gujarat and populations of Spodoptera litura were collected from fields nearby regions. 
The information on sprays occurring in these fields were recorded beforehand, taking help of the local farmers at the time of collecting populations of pest. A mixed culture containing mostly smaller instars like second and third instars were collected in separate bowls along with healthy cotton leaves for survival. Pupae and adults were collected in plastic jars with holes. Tissue papers were kept in these containers and moist conditions were maintained so that the collected culture did not desiccate due to dry conditions. The collection was done in the early morning time. The pest was then reared in laboratory conditions by keeping stringent conditions of temperature and humidity. Incubators were also used, if required to maintain constant conditions for the survival of test insect before testing. The culture was reared for at least three generations so as to ensure the health and infection free nature. After successful rearing, next generation was selected for testing of insecticide monitoring.

Rearing in laboratory conditions: Larvae of Spodoptera litura were reared in controlled laboratory conditions i.e. $25 \pm 2{ }^{\circ} \mathrm{C}, 65-70 \%$ relative humidity and a photoperiod of L: D, 14:10. It was reared on artificial diet (Siddiqui and Dey, 2002). The diet was poured in a plastic container which had partitions in it. The larvae were carefully transferred on diet by using brush. The diet was changed at regular intervals. All the lab paraphernalia used for the whole process was pre-sterilized to avoid fungal and bacterial infections. Until pupation, the larvae were kept on artificial diet. Rearing in container was feasible as there was no cannibalism observed in Spodoptera litura. After complete formation of pupae, they were transferred to bowls. Pupae were also sterilized by using traditional sterilization methods. The completion of pupal stage lead to the beginning of adult emergence. As soon as adult emergence started, healthy male and female adults were released in oviposition pots in the ratio of 2:2. Adult diet was also provided by using honey solution. Moths emerged from the pupae were shifted into glass jars with 1:1 male and female ratio. The moths were provided with water and honey solution. Another method was used for rearing i.e. rearing on natural diet. For this, collection was done for the second time, from the same fields on differenct days. The freshly laid yellow coloured eggs, covered with brown hairs were collected along with the leaves. Adults and larvae of Spodoptera litura were also collected and were bought to the laboratory in perforated polythene bags along with infested leaves. The eggs were kept in Petri dishes (11 $\mathrm{cm}$ dia.) and were covered with fine muslin cloth and secured with rubber bands. The larvae were kept in rearing jars (15 $\mathrm{cm} \times 13 \mathrm{~cm}$ ) covered with muslin cloth and secured with rubber bands. They were daily supplied with fresh cabbage leaves for feeding. The adults were also kept in rearing jars $(15 \mathrm{~cm} \times 13 \mathrm{~cm})$, supplied with a piece of folded paper for oviposition and a cotton swab dipped in $50 \%$ honey solution was hanged from the top in order to provide feeding material for adults. The honey solution was renewed after every 48 hours. The Petri dishes having Spodoptera litura eggs and rearing jars containing larvae and adults were kept in B.O.D. incubator maintained at $27 \pm 2^{\circ} \mathrm{C}$ temperature and $78 \pm 2 \%$ relative humidity. Both types of rearing i.e natural diet and artificial diet was done, to ensure the survival of larvae for the testing against insecticide. The larvae which was reared on natural diet had many challenges like plant health, virus in plant material, fungus development, changing of diet on regular intervals while those reared on artificial diet had different challenges like, diet developing infections, larvae preference for artificial diet. The larvae reared on artificial diet was then selected for testing in subsequent generation.

Insecticide selection: Commercial formulations of Cypermethrin 25EC (Trade name: Auzar, Biostadt India Limited) and Chlorpyriphos 20EC (Trade name: Dursban, Dow Agrosciences), was used for the resistance studies.

Leaf dip bioassay: Test solution was prepared using formulation of Cypermethrin 25EC and Chlorpyriphos 20EC. Healthy cotton leaves were collected from field, washed in laboratory using distilled water. Leaf discs of five centimetres diameter were cut and dipped in the test solutions for ten seconds. On each leaf disc, three 3rd instar larvae (F1 generation) were released, using fine camel hair brush. All the test units were kept in controlled environmental conditions i.e. in B.O.D incubator maintaining temperature and humidity conditions $\left(25 \pm 2^{\circ} \mathrm{C}, 65-70 \%\right)$. Observations were recorded at 96 hours. The live larvae from the first bioassay were reared in separate part of rearing chamber. They were continued on artificial diet to the second generation. The trial was repeated with the same set of treatments and same methodology. This was repeated till five generations.

Data analysis: Larval mortalities were recorded at 96 hours. The larvae were considered dead if they failed to make a coordinated movement when prodded with probe. Data was corrected for control mortalities using Abott's (1925) formula. The data was further analysed by TThe probit analysis method through POLO-PC Program of LeOra, 2003.

\section{RESULTS AND DISCUSSION}

As Table 1 indicates, the mortality observed in fifth generation for Cypermethrin 25EC @ 2, 1, 0.5, 0.25, 0.125 and $0.0625 \mathrm{ppm}$ were $100.00,93.33,46.67,13.33$ and 0.00 $\%$ respectively. If this is compared to the mortalities observed in previous generation, it shows that there is an onset of resistance developed in one of the concentrations i.e. 0.5 ppm as indicated by Fig 1. The LC values observed in all the generations show a gradual development of resistance. As Table 2 indicates LC 50 and LC 90 in first generation for Cypermentrhin 25EC @ 0.5 ppm were 0.43 and 0.82 respectively. In the second generation, these values show a slight increase i.e 0.45 and 0.83 respectively. While in third and fourth generation,these attain a value of $0.45,0.90$ and $0.48,0.92$ respectively. When the bioassay was repeated in 
Table 1: Percent Mortality in in Spodoptera litura when exposed to Cypermethrin.

\begin{tabular}{lccccc}
\hline Treatments & \multicolumn{5}{c}{ \% Mortality } \\
\cline { 2 - 6 } & Generation 1 & Generation 2 & Generation 3 & Generation 4 & Generation 5 \\
\hline Cypermethrin 25EC@ 2 ppm & 100.00 & 100.00 & 100.00 & 100.00 & 100.00 \\
Cypermethrin 25EC @ 1 ppm & 100.00 & 96.67 & 93.33 & 93.33 & 93.33 \\
Cypermethrin 25EC @ 0.5 ppm & 53.33 & 53.33 & 53.33 & 50.00 & 46.67 \\
Cypermethrin 25EC @ 0.25 ppm & 13.33 & 13.33 & 16.67 & 13.33 & 13.33 \\
Cypermethrin 25EC@ 0.125 ppm & 3.33 & 0.00 & 0.00 & 0.00 & 0.00 \\
Cypermethrin 25EC @0.0625 ppm & 0.00 & 0.00 & 0.00 & 0.00 & 0.00 \\
\hline
\end{tabular}

Table 2: LC estimates of Spodoptera litura when exposed to Cypermethrin 25EC.

\begin{tabular}{lccccc}
\hline LC estimates & Generation-1 & Generation-2 & Generation-3 & Generation-4 & Generation-5 \\
\hline LC50 & 0.43 & 0.45 & 0.45 & 0.48 & 0.49 \\
LC90 & 0.82 & 0.83 & 0.90 & 0.92 & 0.94 \\
Slope+- Std Error & $4.51 \pm 0.64$ & $4.86 \pm 0.72$ & $4.31 \pm 0.64$ & $4.50 \pm 0.64$ & $4.48 \pm 0.60$ \\
Chi square & 4.99 & 0.82 & 0.83 & 0.68 & 1.08 \\
Significance & 0.28 & 0.93 & 0.93 & 0.95 & 0.89 \\
\hline
\end{tabular}

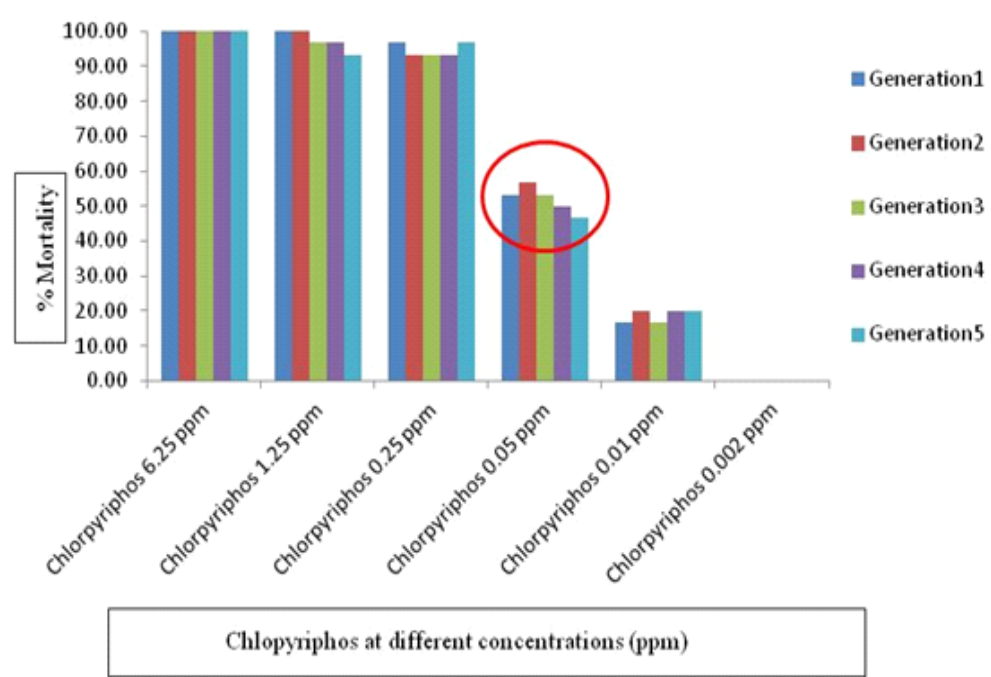

Fig 1: Graph showing resistance developed in Cypermethrin at $0.5 \mathrm{ppm}$.

fifth generation, the LC50 and LC90 values indicate 0.49 and 0.94 respectively. Similarly another insecticide Chlorpyriphos 20EC at different concentrations i.e 6.25, $1.25,0.25,0.05,0.01$ and $0.002 \mathrm{ppm}$ was exposed for five generations. As Table 3 indicates the mortalities obtained in fifth generation were 100, 93.33, 96.67, 46.67 and $20.00 \%$ respectively. The LC 50 and LC 90 values indicated less amount of resistance being developed in the pest.As shown in Table 4 in the fifth generation, LC50 value and LC 90 values were 0.05 and 0.34 . There was an onset of resistance observed in Chlorpyriphos 20EC @ 0.05 as indicated in Fig 2. All these values indicate onset of resistance in both the insecticides in laboratory conditions.

The experimental results indicated greater resistance developed in Cypermehtrin 25EC @ 0.5 ppm (LC 50: 0.49, LC 90: 0.94) as compared to Chlorpyriphos 20EC @ 0.05 ppm. (LC 50: 0.05, LC 90: 0.34). In today's scenario, the farmers are making extensive use of insecticides in lieu of getting fast control of pest attack. But in this process, they become unaware of the fact that resistance is developing slowly and the insecticides which are used extensively will slowly become ineffective. This type of study showed that the insecticides if at all used, must be delivered in an effective rotation pattern based on their respective mode of actions. Though this is difficult to make the farmer understand, it proves to be helpful to the scientists and agriculture professionals worldwide. They can slowly educate the farmer by showing the long term effects of such insecticides used in extensive and haphazard manner. The best alternative to this is switching to organic farming or using bio-pesticides which are having less adverse effect on environment and more positive and healthy effects on plants. The study accounts to discover the long term effects of using the same insecticides on destructive pest like Spodoptera litura. It 
Table 3: Percent Mortality in Spodoptera litura when exposed to Chlorpyriphos 20EC.

\begin{tabular}{lcccrr}
\hline Treatments & \multicolumn{5}{c}{ \% Mortality } \\
\cline { 2 - 6 } & Generation 1 & Generation 2 & Generation 3 & Generation 4 & Generation 5 \\
\hline Chlorpyriphos 6.25 ppm & 100.00 & 100.00 & 100.00 & 100.00 & 100.00 \\
Chlorpyriphos 1.25 ppm & 100.00 & 100.00 & 96.67 & 96.67 & 93.33 \\
Chlorpyriphos 0.25 ppm & 96.67 & 93.33 & 93.33 & 93.33 & 96.67 \\
Chlorpyriphos 0.05 ppm & 53.33 & 56.67 & 53.33 & 50.00 & 46.67 \\
Chlorpyriphos 0.01 ppm & 16.67 & 20.00 & 16.67 & 20.00 & 20.00 \\
Chlorpyriphos 0.002 ppm & 0.00 & 0.00 & 0.00 & 0.00 & 0.00 \\
\hline
\end{tabular}

Table 4: LC estimates of Spodoptera litura when exposed to Chlorpyriphos 20EC.

\begin{tabular}{lccccc}
\hline LC estimates & Generation-1 & Generation-2 & Generation-3 & Generation-4 & Generation-5 \\
\hline LC50 & 0.04 & 0.04 & 0.04 & 0.04 & 0.05 \\
LC90 & 0.17 & 0.19 & 0.27 & 0.28 & 0.34 \\
Slope+- Std Error & $1.99 \pm 0.25$ & $1.79 \pm 0.25$ & $1.62 \pm 0.21$ & $1.56 \pm 0.20$ & $1.47 \pm 0.18$ \\
Chi square & 1.36 & 0.99 & 3.06 & 3.19 & 8.46 \\
Significance & $\mathrm{p}=0.8515$ & $\mathrm{p}=0.9109$ & $\mathrm{p}=0.5485$ & $\mathrm{p}=0.5264$ & $\mathrm{p}=0.0762$ \\
\hline
\end{tabular}

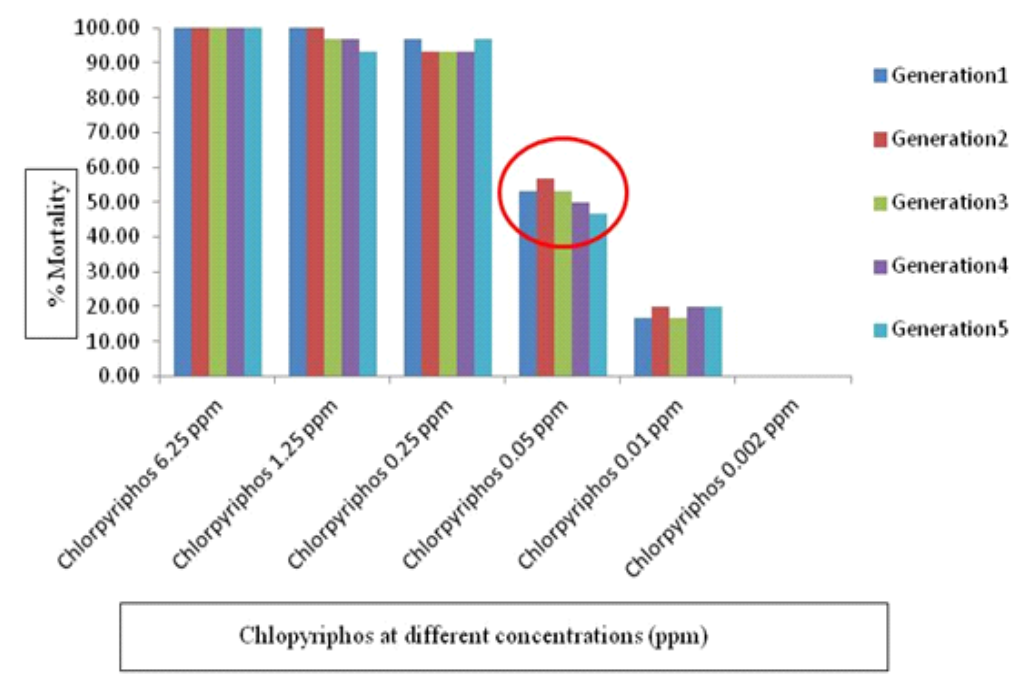

Fig 2: Graph showing resistance developed in Chlorpyriphos at $0.05 \mathrm{ppm}$.

slowly showd onset of resistance in laboratory maintained culture from generation to generation. If usage of insecticides was made, the insecticides must have less amount of stability and more reversion rate. Otherwise it would even cause more problems than providing right solution. This research showed that the insecticides belonging to two different groups must not be used in an uncontrolled manner. They have less stability as indicated by the mortality and LC values. The baseline values may be used for monitoring the resistance development for Spodoptera litura. Thus as discussed in the major findings of this study, we needed to re-think about application of such insecticides which negatively affects the yield, by developing low resistance. Moreover, the effects associated with chemical fertilizers have shown increased inclination of consumers towards organic crops grown by using biofertilizers (Kaur, 2018).

\section{ACKNOWLEDGEMENT}

Thanks are to the Head, Department of Zoology Prof. P.C. Mankodi, for providing Departmental facilities from time to time.

\section{REFERENCES}

Abott, S.W. (1925). A method of computing effectiveness of an insecticide. Journal of Economic Entomology. 18: 265-267.

Ahmed, M., Arif, I.Q. and Ahmed, M. (2007). Occurrence of insecticide resistance in field populations of Spodoptera litura (Lepidoptera: Noctuidae) in Pakistan. Crop Protection. 26: 809-817.

Ahmed, M., Sayyed, A.H., Saleem, M. and Ahmed, M (2008). Evidence for field evolved resistance to newer insecticides in Spodoptera litura (Lepidoptera: Noctuidae) from Pakistan. Crop Protection. 27: 1367-1372. 
Dhir, B.C., Mohapatra S.D., H.K and Senapati, B (1992). Assessment of crop loss in groundnut due to tobacco caterpillar, Spodoptera litura (F.). Indian Journal of Plant Protection. 20: 215-217.

Hiroichi,M. and Naito, A.(1997). Studies on the cold-hardiness and overwintering of Spodoptera litura F. (Lepidoptera: Noctuidae): VI. Possible overwintering areas predicted from meteorological data in Japan. Applied Entomology and Zoology. 32: 167177.

Kaur, R. and Kaur, S. (2018). Biological alternates to synthetic fertilizers: efficiency and future scopes. Indian Journal of Agricultural Research. 52(6): 587-595.

Priscilla, L., Balakrishnan, A., Lalrinsangpuii and Chauhan, A.K. (2017). A Study on the performance of agricultural sector in India. Indian Journal of Agricultural Research. 51(3): 282-286.

Ramakrishnan, N., Saxena, S.V. and resistance in the population of Spodoptera litura (F.) in Andhra Pradesh. Pesticide Science. 18: $23-27$.

Ramasamy, S. (2012). Integrating biopesticides in pest management strategies for tropical vegetable production. Journal of Biopesticides. 5: $36-45$.

Rehan, A., Saleem, M.A. and Freed, S. (2011). Baseline Susceptibility and Stability of Insecticide Resistance of Spodoptera litura (F.) (Lepidoptera: Noctuidae) in the absence of Selection Pressure. Pakistan Zoology Journal. 43(5): 973-978.

Tong, H., Su, Q. and Zhou, X (2013). Field resistance of Spodoptera litura (Lepidoptera: Noctuidae) to organophosphates, pyrethroids, carbamates and four newer chemistry insecticides in Hunan China. Journal of Pesticide Science. 86: 599-609.

Siddiqui, K.H. and Dey, D. (2002). Artificial diets-a tool for insect mass rearing. Jyoti Publishers, New Delhi, India. pp 82-83. 